\title{
ON SATURATED PERMUTATIVE VARIETIES AND CONSEQUENCES OF PERMUTATION IDENTITIES
}

\author{
N. M. KHAN \\ (Received 14 December 1982) \\ Communicated by T. E. Hall
}

\begin{abstract}
We determine which permutative varieties are saturated and classify all nontrivial permutation identities for the class of all globally idempotent semigroups.

1980 Mathematics subject classification (Amer. Math. Soc.): 20 M 07.

Keywords and phrases: semigroup, variety, epimorphism, dominion, saturated, permutation, homotypical, heterotypical.
\end{abstract}

\section{Introduction and summary}

We determine which of the permutative varieties (those admitting nontrivial permutation identities) are saturated. These are precisely those permutative varieties which admit an identity which is not a permutation identity and of which at least one side has no repeated variable. This generalizes a joint result, which determines all saturated commutative varities, of the author [7, Theorem 3.4] and P. M. Higgins [2, Theorem 4]. It has long been known that not all permutative varieties are saturated (see [4, Exercise VII 2(i)]). We also prove a result regarding the consequences of permutation identities, and as applications of this result, we give new and short proofs of all the results of Putcha and Yaqub [10]. Further, an application of our result enables us to classify all nontrivial permutation identities for the class of all globally idempotent semigroups, generalizing Yamada's Theorem 6 of [11]. 


\section{Preliminaries}

Let $U$ be a subsemigroup of a semigroup $S$. We say that $U$ dominates an element $d$ of $S$ if for every semigroup $T$ and for all homomorphisms $\beta, \gamma: S \rightarrow T$, $u \beta=u \gamma$ for all $u$ in $U$ implies $d \beta=d \gamma$. The set of all elements dominated by $U$ is called the dominion of $U$ in $S$ and we denote it by $\operatorname{Dom}_{S}(U)$. It can be easily seen that $\operatorname{Dom}_{S}(U)$ is a subsemigroup of $S$.

A morphism $\alpha: A \rightarrow B$ in the category $C$ of semigroups is called an epimorphism (epi for short) if for all $C \in \mathcal{C}$ and for all morphisms $\beta, \gamma: B \rightarrow C, \alpha \beta=\alpha \gamma$ implies $\beta=\gamma$. The following facts can be easily proved. A morphism $\alpha: S \rightarrow T$ is epi if and only if the inclusion mapping $i: S \alpha \rightarrow T$ is epi, and an inclusion mapping $i: U \rightarrow S$ is epi if and only if $\operatorname{Dom}_{S}(U)=S$.

Following Howie and Isbell [5], we call a semigroup $U$ saturated if $\operatorname{Dom}_{S}(U) \neq$ $S$ for every properly containing semigroup $S$. A variety $\mathcal{T}$ of semigroups will be called saturated if every member of $\mathcal{V}$ is saturated. A variety $\mathcal{V}$ is called epimorphically closed or closed under epis if whenever $\operatorname{Dom}_{S}(U)=S$ for a semigroup $S, U \in \mathcal{V}$ implies $S \in \mathfrak{V}$. Obviously saturated varieties are epimorphically closed, but the converse is not true in general (for example the variety of commutative semigroups is epimorphically closed [6, Corollary 2.5], but not saturated [4, Exercise VII 2(i)]). If $\mathcal{C}$ is a class of semigroups, then every epi from a member of $\mathcal{C}$ is onto if each member of $\mathcal{C}$ is saturated and $\mathcal{C}$ is closed under taking homomorphic images.

Semigroup domains have been characterized by the following celebrated result.

REsult 1 (Isbell's Zigzag Theorem [6, Theorem 2.3] or [4, Theorem VII 2.13]). Let $U$ be any subsemigroup of any semigroup $S$, and let $d$ be any element of $S$. Then $d \in \operatorname{Dom}_{S}(U)$ if and only if $d \in U$ or there are elements $a_{0}, a_{1}, \ldots, a_{2 m} \in U$, $y_{1}, y_{2}, \ldots, y_{m}, x_{1}, x_{2}, \ldots, x_{m} \in S$ such that

$$
\begin{gathered}
d=a_{0} x_{1}, \quad a_{0}=y_{1} a_{1}, \\
y_{i} a_{2 i}=y_{i+1} a_{2 i+1}, \quad a_{2 i-1} x_{i}=a_{2 i} x_{i+1} \quad(i=1,2, \ldots, m-1), \\
a_{2 m-1} x_{m}=a_{2 m}, \quad y_{m} a_{2 m}=d .
\end{gathered}
$$

These equations are called a zigzag of length $m$ over $U$ with value $d$ and spine $a_{0}, a_{1}, \ldots, a_{2 m}$.

Let $X=\left\{x_{1}, x_{2}, \ldots, x_{n}\right\}$ be a set; each element of $X$ will be called a variable. Let $f_{1}\left(x_{1}, x_{2}, \ldots, x_{n}\right)$ and $f_{2}\left(x_{1}, x_{1}, \ldots, x_{n}\right)$ be two words in the variables $x_{1}, x_{2}, \ldots, x_{n}$ (not necessarily containing all the letters $x_{1}, x_{2}, \ldots, x_{n}$ ). Then the pair of words $\left(f_{1}\left(x_{1}, x_{2}, \ldots, x_{n}\right), f_{2}\left(x_{1}, x_{2}, \ldots, x_{n}\right)\right)$ is called an identity in the variables $x_{1}, x_{2}, \ldots, x_{n}$, and is usually written as

$$
f_{1}\left(x_{1}, x_{2}, \ldots, x_{n}\right)=f_{2}\left(x_{1}, x_{2}, \ldots, x_{n}\right) \text {. }
$$


By a permutation identity in the variables $x_{1}, x_{2}, \ldots, x_{n}(n \geqslant 2)$ we mean an identity

$$
x_{1} x_{2} \cdots x_{n}=x_{i_{1}} x_{i_{2}} \cdots x_{i_{n}}
$$

where $\left(i_{1}, i_{2}, \ldots, i_{n}\right)$ is a permutation of the sequence $(1,2, \ldots, n)$. The identities

(C) commutativity $x_{1} x_{2}=x_{2} x_{1}$,

$(L N)$ left normality $x_{1} x_{2} x_{3}=x_{1} x_{3} x_{2}$,

$(R N)$ right normality $x_{1} x_{2} x_{3}=x_{2} x_{1} x_{3}$,

$(N)$ normality $x_{1} x_{2} x_{3} x_{4}=x_{1} x_{3} x_{2} x_{4}$,

are all permutation identities.

An identity $P=Q$ is said to be preserved under epis if for all semigroups $U$ and $S$ such that $U \leqslant S$ and $\operatorname{Dom}_{S}(U)=S, U$ satisfying $P=Q$ implies that $S$ satisfies $P=Q$.

RESULT 2 [8, Theorem 3.1]. All permutation identities are preserved under epis.

RESULT 3. Let $U$ and $S$ be any semigroups with $U$ a subsemigroup of $S$. Take any $d \in S \backslash U$ such that $d \in \operatorname{Dom}_{S}(U)$. Let (1) be a zigzag of shortest possible length $m$ over $U$ with value $d$. Then $x_{j}, y_{j} \in S \backslash U$, for $j=1,2, \ldots, m$.

Proof. Since $d \in S \backslash U$, obviously $x_{1} \in S \backslash U$. So, let us suppose to the contrary that $x_{k} \in U$ for some $k \in\{2, \ldots, m\}$. Then

$$
\begin{gathered}
d=a_{0} x_{1}, \quad a_{0}=y_{1} a_{1}, \\
y_{i} a_{2 i}=y_{i+1} a_{2 i+1}, \quad a_{2 i-1} x_{i}=a_{2 i} x_{i+1} \quad(i=1,2, \ldots, k-2), \\
a_{2 k-3} x_{k-1}=a_{2 k-2} x_{k} \in U, y_{k-1}\left(a_{2 k-2} x_{k}\right)=d,
\end{gathered}
$$

is a zigzag of length $k-1<m$, a contradiction, as required. Similarly $y_{j} \in S \backslash U$ for $j=1,2, \ldots, m$.

RESULT 4. Let $U$ and $S$ be any semigroups with $U$ a subsemigroup of $S$ and $\operatorname{Dom}_{S}(U)=S$. Then for any $d \in S \backslash U$ and any positive integer $k$, there exist $a_{1}, a_{2}, \ldots, a_{k} \in U$ and $d_{k} \in S \backslash U$ such that $d=a_{1} a_{2} \cdots a_{k} d_{k}$. In particular $d \in S^{k}$ for each positive integer $k$.

Proof. Since $d \in S \backslash U$ and $\operatorname{Dom}_{S}(U)=S$, by Result 1, there exist $a_{1} \in U$, $d_{1} \in S \backslash U$ such that $d=a_{1} d_{1}$. Applying again Result 1 , but this time to $d_{1}$, we get $d=a_{1} a_{2} d_{2}$ for some $a_{2} \in U$ and $d_{2} \in S \backslash U$. Continuing this process gives us the required result.

We use whenever possible, and often without comment, the notations and conventions of Clifford and Preston [1] or Howie [4]. 


\section{Some consequences of permutation identities}

An identity $P_{1}=Q_{1}$ is said to be implied by an identity $P_{2}=Q_{2}$ if every semigroup satisfying $P_{2}=Q_{2}$ also satisfies $P_{1}=Q_{1}$.

Proposition 3.1. Let $S$ be any semigroup satisfying (2) with $n \geqslant 3$.

(i) For each $j \in\{2,3, \ldots, n\}$ such that $x_{j-1} x_{j}$ is not a subword of $x_{i_{1}} x_{i_{2}} \cdots x_{i_{n}}, S$ also satisfies the permutation identity

$$
x_{1} x_{2} \cdots x_{j-1} x y x_{j} \cdots x_{n}=x_{1} x_{2} \cdots x_{j-1} y x x_{j} \cdots x_{n} .
$$

(ii) If $x_{1} \neq x_{i_{1}}$ then $S$ also satisfies the permutation identity

$$
x y x_{1} x_{2} \cdots x_{n}=y x x_{1} x_{2} \cdots x_{n} .
$$

Proof. Consider $S^{1}$, and put $x_{0}=1$. Then $S^{1}$ satisfies the condition that $x_{0} x_{1} x_{2} \cdots x_{n}=x_{0} x_{i_{1}} x_{i_{2}} \cdots x_{i_{n}}$ for all $x_{1}, x_{2}, \ldots, x_{n} \in S$. Take any $j \in$ $\{1,2,3, \ldots, n\}$ such that $x_{j-1} x_{j}$ is not a subword of the word $x_{0} x_{i_{1}} x_{i_{2}} \cdots x_{i_{n}}$ (this enables us to prove both statements of the proposition simultaneously).

Let $j=i_{p}$. Then $i_{p-1} \neq j-1$. Let $i_{p-1}=k$. For convenience let us assume that $k<j$ (the case when $k>j$ can be dealt with entirely similarly to the case when $k<j$ ). Define

$$
u\left(x_{0}, x_{1}, x_{2}, \ldots, x_{n}\right)=x_{0} x_{1} x_{2} \cdots x_{n},
$$

and

$$
v\left(x_{0}, x_{1}, x_{2}, \ldots, x_{n}\right)=x_{0} x_{i_{1}} x_{i_{2}} \cdots x_{i_{n}} .
$$

Now take any $x_{1}, x_{2}, \ldots, x_{n}, x, y \in S$. Then

$$
\begin{aligned}
x_{0} x_{1} x_{2} \cdots x_{j-1} x y x_{j} \cdots x_{n} & =u\left(x_{0}, x_{1}, x_{2}, \ldots, x_{j-1} x, y x_{j}, \ldots, x_{n}\right) \\
& =v\left(x_{0}, x_{1}, x_{2}, \ldots, x_{j-1} x, y x_{j}, \ldots, x_{n}\right) \\
& =v\left(x_{0}, x_{1}, \ldots, x_{k} y, \ldots, x_{j-1} x, x_{j}, \ldots, x_{n}\right) \\
& \quad\left(\text { since } i_{p-1}=k \text { and } i_{p}=j\right) \\
& =u\left(x_{0}, x_{1}, \ldots, x_{k} y, \ldots, x_{j-1} x, x_{j}, \ldots, x_{n}\right) \\
& =u\left(x_{0}, x_{1}, \ldots, x_{k} y, \ldots, x_{j-1}, x x_{j}, \ldots, x_{n}\right) \\
& =v\left(x_{0}, x_{1}, \ldots, x_{k} y, \ldots, x_{j-1}, x x_{j}, \ldots, x_{n}\right) \\
& =v\left(x_{0}, x_{1}, \ldots, x_{k}, \ldots ; x_{j-1}, y x x_{j}, \ldots, x_{n}\right) \\
& \quad\left(\text { since } i_{p-1}=k \text { and } i_{p}=j\right) \\
& =u\left(x_{0}, x_{1}, \ldots, x_{j-1}, y x x_{j}, \ldots, x_{n}\right) \\
& =x_{0} x_{1} x_{2} \ldots x_{j-1} y x x_{j} \cdots x_{n}
\end{aligned}
$$

as required, proving both statements of the proposition. 
Remark 1. Proposition 3.1 strengthens Lemmas 19 and 20 of [9].

\section{Epis and permutation identities}

A semigroup $S$ satisfying a nontrivial permutation identity will be called permutative.

Proposition 4.1. Let $U$ be a permutative semigroup and $S$ any semigroup containing $U$ properly such that $\operatorname{Dom}_{S}(U)=S$. Then for any $x, y \in S$ and $s, t \in$ $S \backslash U$,

$$
s x y t=s y x t .
$$

Proof. Since $U$ is permutative, by Result $2, S$ is also permutative. Therefore, by Proposition 3.1 there exist $n$ and $j \in\{1,2, \ldots, n\}$ such that $S$ also satisfies the following permutation identity

$$
x_{1} x_{2} \cdots x_{j-1} x y x_{j} \cdots x_{n}=x_{1} x_{2} \cdots x_{j-1} y x x_{j} \cdots x_{n}
$$

(when $j=1$, we assume that the word $x_{1} x_{2} \cdots x_{j-1}$ is the empty word).

Since, by Result 4, for all $s, t \in S \backslash U$ we have $s, t \in S^{k}$ for all positive integers $k$, the result now follows.

REMarK 2. Proposition 4.1 can also be proved by appealing to [10, Theorem 1] and Result 2.

The proof of Proposition 4.1 could be easily modified to give the following corollaries. In Corollaries 4.3 and 4.4 bracketed statements are dual to the other statements.

In results 4.2 to $4.7, U$ is any semigroup satisfying (2), and $S$ is any semigroup containing $U$ properly and such that $\operatorname{Dom}_{S}(U)=S$.

COROLlaRY 4.2. If (2) is nontrivial, then $s x_{1} x_{2} \cdots x_{k} t=s x_{j_{1}} x_{j_{2}} \cdots x_{j_{k}} t$ for all $s, t \in S \backslash U, x_{1}, x_{2}, \ldots, x_{k} \in S$, and for any permutation $j$ of the set $\{1,2, \ldots, k\}$.

CoROllaRy 4.3. If $i_{1} \neq 1\left[i_{n} \neq n\right]$, then $x y t=y x t[s x y=s y x]$ for all $x, y \in S$ and $t \in S \backslash U[s \in S \backslash U]$.

Corollary 4.4. If $i_{1} \neq 1\left[i_{n} \neq n\right]$, then

$$
x_{1} x_{2} \cdots x_{k} t=x_{j_{1}} x_{j_{2}} \cdots x_{j_{k}} t \quad\left[s x_{1} x_{2} \cdots x_{k}=s x_{j_{1}} x_{j_{2}} \cdots x_{j_{k}}\right] \text {, }
$$

for all $x_{1}, x_{2}, \ldots, x_{k} \in S, t \in S \backslash U[s \in S \backslash U]$, and for any permutation $j$ of the set $\{1,2, \ldots, k\}$. 
Proposition 4.5. If $i_{1} \neq 1$ and either $i_{n-1} \neq n-1$ or $i_{n} \neq n$, then $x y z=y x z$ for all $x, z \in S$ and $y \in S \backslash U$.

Proof. Since $y \in S \backslash U$, we may let (1) be a zigzag of shortest possible length $m$ over $U$ with value $y$. Then

$$
\begin{aligned}
x y z & =x a_{0} x_{1} z \\
& =a_{0} x x_{1} z \quad\left(\text { by Corollary 4.3, since } i_{1} \neq 1 \text { and } x_{1} \in S \backslash U\right) \\
& =y_{1} a_{1} x x_{1} z \\
& =y_{1} x a_{1} x_{1} z \quad\left(\text { by Corollary } 4.4, \text { since } i_{1} \neq 1 \text { and } x_{1} \in S \backslash U\right) \\
& =y_{1} x a_{2} x_{2} z \\
& =y_{1} a_{2} x x_{2} z \quad\left(\text { by Corollary } 4.4, \text { since } i_{1} \neq 1 \text { and } x_{2} \in S \backslash U\right) \\
& =y_{2} a_{3} x x_{2} z=\cdots=y_{m} a_{2 m-1} x x_{m} z \\
& =y_{m} x a_{2 m-1} x_{m} z \quad\left(\text { by Corollary } 4.4, \text { since } i_{1} \neq 1 \text { and } x_{m} \in S \backslash U\right) \\
& =y_{m} x a_{2 m} z \\
& =y_{m} a_{2 m} x z \quad\left(\begin{array}{l}
\text { by Proposition } 3.1 \text { if } i_{n-1} \neq n-1 \text { and } i_{n}=n \text { since } \\
y_{m} \in S^{n-1}, \text { otherwise by Corollary } 4.4 \text { since } y_{m} \in S \backslash U
\end{array}\right) \\
& =y x z, \quad
\end{aligned}
$$

as required.

We give a corollary to Proposition 4.5 and its dual.

COROLlary 4.6. If $i_{1} \neq 1$ and $i_{n} \neq n$, then $s_{1} s_{2} s_{3}=s_{j_{1}} s_{j_{2}} s_{j_{3}}$ for any $s_{1}, s_{2}, s_{3} \in S$ with one or more being in $S \backslash U$, and for any permutation $j$ of the set $\{1,2,3\}$.

COROLlaRY 4.7. If $i_{1} \neq 1$ and either $i_{n-1} \neq n-1$ or $i_{n} \neq n$, then

$$
s_{1} s_{2} \cdots s_{k}=s_{j_{1}} s_{j_{2}} \cdots s_{j_{k-1}} s_{k}
$$

for any $s_{1}, s_{2}, \ldots, s_{k} \in S$ such that $s_{q} \in S \backslash U$ for some $q \in\{1,2, \ldots, k-1\}$ and for any permutation $j$ of the set $\{1,2, \ldots, k-1\}$.

Proof. We have

$$
\begin{aligned}
s_{1} s_{2} \cdots s_{q} \cdots s_{k} & =s_{1} s_{2} \cdots s_{q-1} s_{q+1} \cdots s_{k-1} s_{q} s_{k} \quad \text { (by Proposition 4.5) } \\
& =s_{j_{1}} s_{j_{2}} \cdots s_{j_{l-1}} s_{j_{l+1}} \cdots s_{j_{k-1}} s_{q} s_{k} \\
& \quad \text { (where } s_{q}=s_{j_{i}}, \text { by Corollary 4.4) } \\
& =s_{j_{1}} s_{j_{2}} \cdots s_{j_{l-1}} s_{q} s_{j_{l+1}} \cdots s_{j_{k-1}} s_{k} \quad \text { (by Proposition 4.5) } \\
& =s_{j_{1}} s_{j_{2}} \cdots s_{j_{k-1}} s_{k} \text { as required. }
\end{aligned}
$$




\section{Saturated permutative varieties}

A variety $\mathcal{T}$ admitting a nontrivial permutation identity is called permutative.

THeOREM 5.1. Let $U$ be a permutative semigroup. Then $U$ is saturated if $U$ satisfies an identity I such that (i) $I$ is not a permutation identity, and (ii) at least one side of I has no repeated variable.

Proof. To prove the theorem, we can assume without loss of generality, which we prove below, that $I$ has the form

$$
x_{1} x_{2} \cdots x_{m}=w\left(x_{1}, x_{2}, \ldots, x_{m}\right)
$$

where $\left|x_{i}\right|_{w} \geqslant 1$ for $i=1,2, \ldots, m$, and $\left|x_{j}\right|_{w} \geqslant 2$ for some $j \in\{1,2, \ldots, m\}\left(|x|_{w}\right.$ for any variable $x$ is the number of occurrences of the variable $x$ in the word $w$ ).

For if $I$ is homotypical, as $I$ is nonpermutative, $I$ has to be of the form (3). So let us assume next that $I$ is not homotypical. Then $I$ has one of the following three forms:

(i) $x_{1} x_{2} \cdots x_{m}=f\left(x_{1}, x_{2}, \ldots, x_{m}, x_{m+1}, \ldots, x_{m+k}\right)$ where $f$ is some word in the variables $x_{1}, \ldots, x_{m+k}$ such that $k>0$, and $\left|x_{i}\right|_{f} \geqslant 1, i=1,2, \ldots, m+k$;

(ii) $x_{1} x_{2} \cdots x_{m}=f\left(x_{1}, x_{2}, \ldots, x_{m}\right)$ where $f$ is some word in the variables $x_{1}, \ldots, x_{m}$ such that for some $i, j \in\{1,2, \ldots, m\},\left|x_{i}\right|_{f}=0$ and $\left|x_{j}\right|_{f} \geqslant 1$;

(iii) $x_{1} x_{2} \cdots x_{m}=f\left(x_{1}, \ldots, x_{m}, x_{m+1}, \ldots, x_{m+k}\right), k>0$, where $f$ is some word in $x_{1}, x_{2}, \ldots, x_{m+k}$ such that for some $i \in\{1,2, \ldots, m\},\left|x_{i}\right|_{f}=0$, and $\left|x_{m+j}\right| \geqslant 1$ for $j=1, \ldots, k$.

Now in case (i), by replacing the variables $x_{m+1}, \ldots, x_{m+k}$, if necessary, by $x_{j}$ for some $j \in\{1,2, \ldots, m\}$, we immediately get an identity of the form (3).

In case (ii), let $j \in\{1,2, \ldots, m\}$ be such that $x_{j} \notin C(f)$ (the content of $f$ ). Now by replacing the variable $x_{j}$ by $x_{j}^{2}$ we get that $S$ satisfies the identity $x_{1} x_{2} \cdots x_{m}$ $=x_{1} x_{2} \cdots x_{j-1} x_{j}^{2} x_{j+1} \cdots x_{m}$ (since the R.H.S. of $I$ is independent of the choice of the variable $x_{j}$ ) which is of the form (3) above.

Finally in the last case we can get an identity of the form (3) simply by applying the techniques of the above two cases.

To prove the theorem let us now assume to the contrary that $U$ is not saturated. Therefore there exists a semigroup $S$ containing $U$ properly such that $\operatorname{Dom}_{S}(U)$ $=S$.

LEMMA 5.2. For all $a \in U, x, y \in S \backslash U$, and for some $q \geqslant 2$

$$
x a y=x a^{q} y \text {. }
$$

Proof. Since $y \in S \backslash U$, from Result 4, we have

$$
y=a_{1} a_{2} \cdots a_{m} y_{m}
$$


for some $y_{m} \in S \backslash U$ and $a_{1}, a_{2}, \ldots, a_{m} \in U$. Now

$$
\begin{aligned}
x a y & =x a a_{1} a_{2} \cdots a_{m} y_{m} \\
& =x a_{1} a_{2} \cdots\left(a a_{j}\right) \cdots a_{m} y_{m} \quad(\text { by Corollary 4.2) } \\
& =x w\left(a_{1}, a_{2}, \ldots, a a_{j}, \ldots, a_{m}\right) y_{m} \\
& \left.=x a^{q} w\left(a_{1}, a_{2}, \ldots, a_{j}, \ldots, a_{m}\right) y_{m} \quad \text { (by Corollary } 4.2, \text { where } q=\left|x_{j}\right|_{w} \geqslant 2\right) \\
& =x a^{q} a_{1} a_{2} \cdots a_{m} y_{m}=x a^{q} y, \quad \text { as required. }
\end{aligned}
$$

We give a corollary to the proof of Lemma 5.2 .

Corollary 5.3. For all $a \in U, s, t \in S^{1}$ and $x, y \in S \backslash U, x s a t y=x s a^{q} t y$ for some $q \geqslant 2$.

Now to complete the proof of Theorem 5.1, we take any $d \in S \backslash U$, and let (1) be a zigzag for $d$ of shortest possible length $m$ over $U$. Then

$$
\begin{aligned}
d & =y_{1} a_{1} x_{1}=y_{1} a_{1}^{q} x_{1} \quad \text { (by Result } 3 \text { and Lemma 5.2) } \\
& =y_{1} a_{1}^{q-1} a_{2} x_{2} \\
& =y_{1} a_{2} a_{1}^{q-1} x_{2} \quad \text { (by Corollary 4.2) } \\
& =y_{2} a_{3} a_{1}^{q-1} x_{2} \\
& =y_{2} a_{1}^{q-1} a_{3} x_{2} \quad \text { (by Corollary 4.2) } \\
& =y_{2} a_{1}^{q-1} a_{3}^{q} x_{2} \quad(\text { by Corollary 5.3) } \\
& \vdots \\
& =y_{m} a_{1}^{q-1} a_{3}^{q-1} \cdots a_{2 m-3}^{q-1} a_{2 m-1}^{q} x_{m} \\
& =y_{m} a_{1}^{q-1} a_{3}^{q-1} \ldots a_{2 m-1}^{q-1} a_{2 m-1} x_{m} \\
& =y_{m} a_{2 m-1} a_{1}^{q-1} a_{3}^{q-1} \cdots a_{2 m-1}^{q-2} a_{2 m-1} x_{m} \\
& =y_{m-1} a_{2 m-2} a_{1} a_{3} \cdots a_{2 m-3} w^{\prime} a_{2 m-1} x_{m}
\end{aligned}
$$

(by Corollary 4.2 , where $w^{\prime}=a_{1}^{q-2} a_{3}^{q-2} \cdots a_{2 m-1}^{q-2}$ )

$$
\begin{aligned}
& =y_{1} a_{2} a_{4} \cdots a_{2 m-2} a_{1} w^{\prime} a_{2 m-1} x_{m} \\
& =y_{1} a_{1} a_{2} a_{4} \cdots a_{2 m-2} w^{\prime} a_{2 m-1} x_{m} \\
& =a_{0} a_{2} a_{4} \cdots a_{2 m-2} w^{\prime} a_{2 m} \in U,
\end{aligned}
$$

a contradiction. This completes the proof of the theorem.

The next theorem characterizes all permutative varieties which are saturated, and thus provides a generalization of Theorem 3.4 of [7] from commutative varieties to permutative varieties. 
THEOREM 5.4. A permutative variety is saturated if and only if it admits an identity I such that

(i) I is not a permutation identity, and

(ii) at least one side of $I$ has no repeated variable.

Proof. The 'if statement' of the theorem follows from Theorem 5.1. So it remains only to show that any saturated permutative variety $\mathcal{V}$ has to admit an identity $I$ of the above form. Now take any saturated permutative variety $\mathcal{}$, and suppose to the contrary that $\mathfrak{V}$ does not admit any identity $I$ of the above form. Therefore all the identities of $\mathfrak{V}$ are either permutation identities or of the type whose both sides have repeated variables. Since the semigroup $U$ of [2] is commutative, it satisfies all identities of $\mathcal{V}$ by [2, Lemma 3], whence $U \in \mathcal{V}$. As $U$ is not saturated, $\mathcal{V}$ cannot be saturated, a contradiction. This completes the proof of the theorem.

REMARK 3. P. M. Higgins has independently shown with a different technique [3, Corollary to Theorem 19] that if a variety $\mathfrak{V}$ admits an identity of the form given in the statement of the Theorem 5.4 and a permutation identity (2) such htat $i_{1} \neq 1$ and $i_{n} \neq n$, then $\mathcal{V}$ is saturated.

\section{On applications of Proposition 3.1.}

A semigroup $S$ is said to be medial if it satisfies the normality identity $(N)$. Below we give direct and short proofs of all the results of Putcha and Yaqub [10] and strengthen them by reducing the number of factors that are needed in each case.

Proposition 6.1. Let $S$ be a semigroup. Take any integers $m \geqslant 0, p \geqslant 0, n \geqslant 2$. If $S$ satisfies the permutation identity

$$
x_{1} x_{2} \cdots x_{m} y_{1} y_{2} \cdots y_{n} z_{1} z_{2} \cdots z_{p}=x_{1} x_{2} \cdots x_{m} y_{i_{1}} y_{i_{2}} \cdots y_{i_{n}} z_{1} z_{2} \cdots z_{p}
$$

where $i_{1} \neq 1$ and $i_{n} \neq n$, then for all $k \geqslant 2 n, S$ also satisfies any permutation identity of the form

$$
x_{1} x_{2} \cdots x_{m} y_{1} y_{2} \cdots y_{k} z_{1} z_{2} \cdots z_{p}=x_{1} x_{2} \cdots x_{m} y_{j_{1}} y_{j_{2}} \cdots y_{j_{k}} z_{1} z_{2} \cdots z_{p}
$$

for any permutation $j$ of the set $\{1,2, \ldots, k\}$.

PRoof. Since $i_{1} \neq 1$ and $i_{n} \neq n, x_{m} y_{1}, y_{n} z_{1}$ and $y_{q-1} y_{q}$ for some $q \in\{2,3, \ldots, n\}$ are not subwords of $x_{1} x_{2} \cdots x_{m} y_{1} y_{2} \cdots y_{n} z_{1} z_{2} \cdots z_{p}$. The proof now follows from repeated applications of Proposition 3.1 to the word $x_{1} x_{2} \cdots x_{m} y_{1} y_{2}$ $\cdots y_{k} z_{1} z_{2} \cdots z_{p}$. This is so because we can commute any adjacent pair of $y_{i}$ 's, by 
Proposition 3.1, if we have the following:

(i) $m$ elements to the left of the pair and $(n+p)$ to the right, or

(ii) $(m+n)$ elements to the left of the pair and $p$ to the right, or

(iii) $(m+q-1)$ elements to the left and $(n+p-q+1)$ to the right.

Since $k \geqslant 2 n$, at least one of the cases (i), (ii) and (iii) will always occur.

Remark 4. Proposition 6.1 implies Lemma 1 and Lemma 2 of [10], and gives a much smaller value for $k$ than that given in [10].

COROLlaRY 6.2. Let $S$ be any semigroup satisfying (2) with $n \geqslant 3$ and with $i_{1} \neq 1$ and $i_{n} \neq n$. Then for all integers $k \geqslant 2 n, S$ satisfies every permutation identity in $k$ variables.

The following proposition is easily proved from Proposition 3.1.

Proposition 6.3. Let $S$ be any semigroup satisfying (2) with $n \geqslant 3$. Then for each $j \in\{2,3, \ldots, n\}$ such that $x_{j-1} x_{j}$ is not a subword of $x_{i_{1}} x_{i_{2}} \cdots x_{i_{n}}$, for all $m \geqslant j-1$, $p \geqslant n-j+1$ and for all $u \in S^{m}, v \in S^{p}$ we have $u x_{1} x_{2} v=u x_{2} x_{1} v$ for all $x_{1}, x_{2} \in S$. In particular $S^{K}$ is medial for all $k \geqslant \max (j-1, n-j+1)$.

Remark 5. As corollaries to Corollary 6.2 and Proposition 6.3, we get Theorem 2 and 1 of $[10]$ respectively.

Let $\Omega$ be any class of semigroups. Let $P_{1}=P_{2}$ and $Q_{1}=Q_{2}$ be permutation identities. Then $P_{1}=P_{2}$ and $Q_{1}=Q_{2}$ are said to be equivalent with respect to $\Omega$ if every semigroup of $\Omega$ satisfying $P_{1}=P_{2}$ also satisfies $Q_{1}=Q_{2}$ and vice-versa.

A semigroup $S$ is globally idempotent if for every a $\in \mathrm{S}$ there exist $x, y \in S$ such that $a=x y$, that is, if $S=S^{2}$.

THEOREM 6.4. Let $\Omega$ be the class of globally idempotent semigroups. Let

$$
x_{1} x_{2} \cdots x_{n}=x_{i_{1}} x_{i_{2}} \cdots x_{i_{n}}
$$

be any nontrivial permutation identity. Then (4) is equivalent with respect to $\Omega$ to

(i) commutativity if $i_{1} \neq 1$ and $i_{n} \neq n$,

(ii) left normality if $i_{1}=1$ and $i_{n} \neq n$,

(iii) right normality if $i_{1} \neq 1$ and $i_{n}=n$,

(iv) normality if $i_{1}=1$ and $i_{n}=n$.

Proof. Suppose that a globally idempotent semigroup $S$ satisfies (4).

(i) Take any $x, y \in S$. Since $S$ is globally idempotent, for any $m \geqslant 1$ we have $y=a b$ for some $a, b \in S^{m}$. 
Now by Proposition 3.1, we have

$$
\begin{aligned}
x y & =x a b \\
& =a x b \quad\left(\text { since } i_{1} \neq 1\right) \\
& =a b x \quad\left(\text { since } i_{n} \neq n\right) \\
& =y x .
\end{aligned}
$$

Hence $S$ satisfies commutativity.

(ii) Take any $x, y, z \in S$. Since $x \in S^{n}$, and $i_{n} \neq n$, by Proposition 3.1, we have $x y z=x z y$.

(iii) This is the dual statement of statement (ii).

(iv) Take any $x, y, z, w \in S$. Since $S$ is globally idempotent $x, y, z, w \in S^{n}$. Now, since $n \geqslant \max (j-1, n-j+1)$ for any $j$ such that $x_{j-1} x_{j}$ is not a subword of $x_{i_{1}} x_{i_{2}} \cdots x_{i_{n}}$, from Proposition 6.2 we have that $x y z w=x z y w$.

The converse implications are obvious.

Remark 6. As a corollary to Theorem 6.4, we have Theorem 6 of Yamada [11]. Since commutativity, left normality, right normality and normality are nonequivalent to each other with respect to the class of bands, they are also nonequivalent to each other with respect to the class of globally idempotent semigroups.

\section{Acknowledgment}

I thank Tom Hall, my supervisor, for his sustained help and various helpful suggestions throughout the preparation of this paper.

\section{References}

[1] A. H. Clifford and G. B. Preston, The algebraic theory of semigroups, Math. Surveys 7, Amer. Math. Soc., Providence, R.I., vol. I; 1961, vol. II, 1967.

[2] P. M. Higgins, 'The commutative varieties of semigroups for which epis are onto', Proc. Edinburgh Math. Soc., to appear.

[3] P. M. Higgins, 'Saturated and epimorphically closed varieties of semigroups', J. Austral. Math. Soc, to appear.

[4] J. M. Howie, An introduction to semigroup theory (London Math. Soc. Monographs 7, Academic Press, 1976).

[5] J. M. Howie and J. R. Isbell, 'Epimorphisms and dominions II', J. Algebra 6 (1967), 7-21.

[6] J. R. Isbell, 'Epimorphisms and dominions', Proceedings of the Conference on Categorical Algebra, La Jolla, 1965, pp. 232-246 (Lange and Springer, Berlin, 1966).

[7] N. M. Khan, 'Epimorphisms, dominions and varities of semigroups', Semigroup Forum 25 (1982), 331-337.

[8] N. M. Khan, 'Epimorphically closed permutative varities', submitted. 
[9] P. Perkins, 'Bases for equation theories of semigroups'. J. Algebra 11 (1969), 298-314.

[10] M. S. Putcha and A. Yaqub, 'Semigroups satisfying permutation identities', Semigroup Forum 3 (1971), 68-73.

[11] M. Yamada, 'Regular semigroups whose idempotents satisfy permutation identities', Pacific $J$. Math. 21 (1967), 371-392.

Department of Mathematics

Monash University

Clayton, Victoria

Australia 3168 